\title{
Navier Solution for Buckling Analysis of Size-Dependent Functionally Graded Micro-Plates
}

\section{Abstract}

In the present paper, buckling analysis of functionally graded rectangular micro-plates, on the basis of strain gradient theory with one length scale parameter is studied. Considering the Kirchhoff plate theory and the principle of minimum total potential energy, governing equations of micro-plate subjected to in-plane loads are extracted. In accordance with functionally graded distribution of material properties through the thickness, higher order governing equation of sixth order is derived. Consequently, the stability equation is solved analytically for simply supported micro-plates and the effects of material properties, micro-structure parameters, dimensions and loading conditions are expounded on the critical buckling load. Developing the strain gradient theory for buckling analysis of micro-plates made of functionally graded material is a significant novelty of the presented study.

\section{Keywords}

Rectangular micro-plate, Strain gradient theory, Buckling analysis, Functionally graded, Kirchhoff plate theory, Navier solution.

\section{H. Farahmand ${ }^{a}{ }^{*}$}

S. S. Naseralavi ${ }^{\text {b }}$

A. Iranmanesh ${ }^{\text {b }}$

M. Mohammadi ${ }^{\mathrm{c}}$

${ }^{a}$ Department of Mechanical Engineering, Kerman Branch, Islamic Azad University, Kerman, Iran.

b Department of Civil Engineering, Vali-e-Asr University of Ranfasjan, Rafsanjan, Iran.

c Young Researchers and Elites Club,

Kerman Branch, Islamic Azad

University, Kerman, Iran.

* Corresponding author email:

hamed_1256@yahoo.com

http://dx.doi.org/10.1590/1679-78252554

Received 18.10.2015

In revised form 21.09.2016

Accepted 20.10.2016

Available online 31.10.2016

\section{INTRODUCTION}

Increasing demands of Micro Electro Mechanical Systems (MEMS) and Nano Electro Mechanical Systems (NEMS) in different fields, especially in bio-mechanics and smart systems, have attracted great numbers of researches toward these subjects. NEMS and MEMS usually are made out of different parts which can be modeled as micro beams or plates. For these models, the most import case of mechanical study is stability analysis. Therefore, buckling analysis of these structures is so important because in the bifurcation point, they buckle and become unstable. 
Experimental studies on the plates and beams show the inevitable effect of small scale in micro and nano structures (Chong and Lam (1999), Lakes (1980) and (1986), Yang and Lakes (1982), Hofstetter et al. (2005)). Hence, utilizing classical theories (Wang et al. (2000)) for analyzing the members such as plates are modified depending on different proposed models. For investigation of micro-plates, different theories have been proposed by the researchers, e.g. strain gradient theory (Mindlin (1964), Mindlin and Eshel (1968)), couple stress theory (Toupin (1962)) and micro polar theory (Eringen (1966) and (1967)). In these theories, the effects of micro-structures and the size effects are considered in constitutive equations.

Among the proposed theories, strain gradient theory is one of the most usable one which is a higher order generalized form of classical elasticity theory. According to the general form of Mindlin theory (Mindlin (1964)), strain energy density is composed of three terms. These terms are gradients of displacements, gradients of strain and gradients of rotation, respectively. In the couple stress theory, it is assumed that the energy density contain expressions of gradient of rotation and the conjugated stress. Mindlin and Eshel (1968) considered the first order gradient of strains in the energy density and proposed a form contains five elastic constants in addition to two usual Lam e' constants for an isotropic linear elastic micro structured solid.

A review of the above mentioned higher order theories of elasticity can be found in the works of Tiersten and Bleustein(1974), Vardoulakis and Sulem (1995), Lakes (1995), Papargyri-Beskou and Beskos (2008).

A size dependent model for thin micro-plates (Kirchhoff micro-plates) has been presented by Farahmand et al. (2011) and Wang et al. (2011). They considered length scale parameters to capture the effect of size on the solution and improved the strain gradient theory in order to predict results for micro-plates. Papargyri-Beskou and Beskos (2008) investigated analytical solution for buckling analysis of strain gradient elastic thin micro-plates based on the Kirchhoff theory. The variational approach was utilized to determine the governing equations and boundary conditions in Lazopoulos work(2009).

Since MEMS and NEMS components are usually subjected to the thermal effects (because of electrical resistance), applying heat resistant materials in construction of their components is a challenging research topic in engineering. Recent development in the metallurgy engineering leads to suggest new materials which combine different layers (composites) with continuous distribution of material properties. Functionally graded (FG) distribution of materials was the proposed solution for the problem (Kiozumi (1997)). Functionally graded materials (FGMs) are usually combination of ceramic and metal. Beside the important mechanical properties of the metal part, the ceramic components in FGMs improve thermal capacity of the composite. Therefore, FGMs can be considered as suitable materials for micro-structures in variety of their applications, especially in MEMS. Several studies on the application of functionally graded materials for classical plate models were done (Mohammadi et al.(2009) and (2010)). Thinh et al. (2016) studied buckling and vibration analysis of functionally graded plates on the basis of eight unknown higher order shear deformation theory. They used Hamilton's principle for determining the equation of motion. Wu et al. (2013) developed the unified formulations of finite cylindrical layer methods (FCLMs) based on the Reissner mixed variational theorem (RMVT) and the principle of virtual displacements (PVD) for the three-dimensional (3D) linear buckling analysis of simply-supported, multilayered functionally graded material (FGM) circular hollow 
cylinders and laminated composite ones under combined axial compression and external pressure. Buckling analysis of functionally graded rectangular plates based on the Kirchhoff plate theory was carried by Ramu and Mohanty (2014). Finite element method was used for analysis of plate subjected to uniaxial and biaxial distributed loads on the simply supported edges.

Some studies were done on the micro-beams made out of FGMs, e.g., Ke and Wang (2011) investigated the dynamic stability of micro-beams made out of FG materials. They used the modified couple stress theory for analyzing a Timoshenko beam. It was assumed that material properties of the FG micro-beam fluctuate through the thickness, and that the material properties were estimated by Mori-Tanaka homogenization technique. Consequently, the effects of length scale parameter and material properties on the dynamic stability of the beam were then presented.

Based on the modified couple stress theory, a new size dependent formulation for the functionally graded Timoshenko beam was derived by Asghari et al. (2011). In that study, material properties were considered to vary through the thickness by power law distribution. The static and vibration responses of cantilever and simply supported beams were determined for different length scale parameters. Given the fluctuation of material properties in thickness direction, according to power law function, nonlinear vibration of size dependent functionally graded Timoshenko micro-beams was done by Ke et al. (2012).

Modified couple stress theory of FG Timoshenko micro-beams, in addition to von Karman geometric nonlinearity were used in their study to obtain higher order nonlinear governing equations. Moreover, different boundary conditions are surveyed by using the Hamilton principle in their work.

As it was reviewed briefly, most of the studies on the FG micro-structures have been limited to beams not plates. Hence, in the present study, buckling analysis of thin functionally graded rectangular micro-plates is presented. In accordace to the strain gradient theory along with inclusion of one length scale, governing equations are obtained for flextural rectangular micro-plate. Eventually, the effects of length scale parameter and material properties on the critical buckling loads of microplate are investigated in detail.

\section{STRAIN GRADIENT RLSTICITY THEORY}

In micro-structures as the result of size effects, micro-structural effects should be considered in equations. According to the strain gradient theory, micro-structural effects are encountered by stress-strain relations in generalized form as follows

$$
\sigma=f\left(\sigma_{0}, \varepsilon, g^{n}, \eta\right)
$$

where $\sigma_{0}$ is initial stress, $\varepsilon$ is strain, $g$ is length scale parameter and $\eta$ is strain gradient. The simplest possible version of strain gradient elasticity theory based on the Mindlin studies (Mindlin (1964), Mindlin and Eshel (1968)), (which contains five length scale constants in addition to the two Lam é constants) is a model with just one length scale constant in addition to the Lam é constants. The constitutive equations for this model are given as (Yang et al. (2002)):

$$
\begin{aligned}
& \bar{\sigma}=\bar{\tau}-\nabla \cdot \bar{\mu} \\
& \bar{\tau}=2 \mu \bar{\varepsilon}+\lambda \operatorname{tr}(\bar{\varepsilon}) I \\
& \bar{\mu}=g^{2} \nabla \bar{\tau}=g^{2} \lambda(\nabla \operatorname{tr} \bar{\varepsilon}) I+2 g^{2} \mu \nabla \bar{\varepsilon}
\end{aligned}
$$


In the above equations, $\bar{\sigma}$ and $\bar{\tau}$ are the total and the classical Cauchy stress tensors, respectively. Also, parameter $I$ is the unit tensor, $\bar{\varepsilon}$ and $\operatorname{tr}(\bar{\varepsilon})$ are the strain tensor and its trace which are expressed in terms of the displacement vector $\mathbf{u}$ as:

$$
\begin{aligned}
& \bar{\varepsilon}=\frac{1}{2}\left(\nabla \mathbf{u}+\nabla \mathbf{u}^{T}\right) \\
& \operatorname{tr}(\bar{\varepsilon})=\nabla . \mathbf{u}
\end{aligned}
$$

In Eq. (2), $g^{2}$ is the volumetric strain energy gradient coefficient or simply gradient coefficient where $g$ (length scale parameter) is defined as the internal or characteristic length of a microstructure. Also $\lambda$ and $\mu$ are the two classical Lam é constants.

Imposing the strain gradient term with length scale parameter in conventional elasticity as a constraint was discussed by Aifantis (1999) and Farahmand and Arabnejad (2010). Comparison of experimental results from torsion and bending tests of beams with the theoretical ones obtained from the study and other higher-order elasticity models reveal that magnitude of the gradient coefficient $g$ (internal length) is of the same order as the diameter of the basic building block in a microstructure, e.g. the grain in metals or ceramics, the osteon in bones or the cell in foams (Farahmand and Arabnejad (2010)).

\section{GOVERNING EQUATIONS OF FUNCTIONALLY GRADED MICRO-PLATE}

Consider a thin flat rectangular micro-plate subjected to in-plane load as shown in Figure 1, where the $z$ coordinate is measured through the thickness direction, and variables $x$ and $y$ are in-plane coordinate.

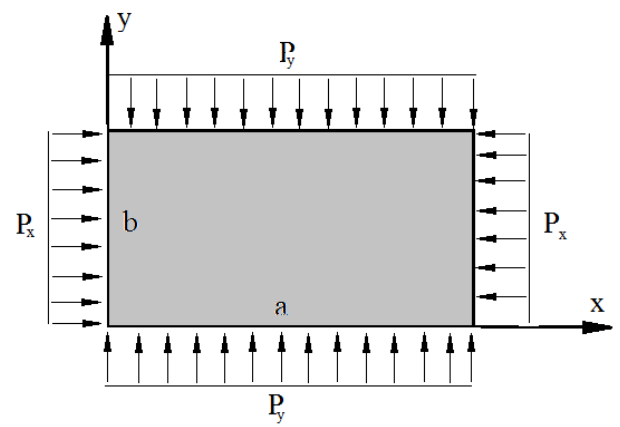

Figure 1: Rectangular micro-plate subjected to in-plane distributed load.

Based on the classical (Kirchhoff) plate theory (CPT), the displacement field is expressed as metal

$$
u_{x}(x, y, z)=u-z w_{, x} \quad u_{y}(x, y, z)=v-z w_{, y} \quad u_{z}(x, y, z)=w(x, y)
$$

where $u_{x}$ and $u_{y}$ are in-plane displacement in $x$ and $y$ directions, respectively and $u_{z}$ is the transverse displacement. Also, $u$ and $v$ are mid-plane displacements and (,) indicates differentia- 
tion with respect to the variables. Considering Von-Karman hypothesis for linear terms, the kinematic relations of micro-plate are written as

$$
\varepsilon_{x x}=\bar{\varepsilon}_{x}+z k_{x} \quad \varepsilon_{y y}=\bar{\varepsilon}_{y}+z k_{y} \quad \gamma_{x y}=2 \varepsilon_{x y}=\bar{\varepsilon}_{x y}+z k_{x y}
$$

In the above equations, strain and curvature components are defined as

$$
\begin{array}{lll}
\bar{\varepsilon}_{x}=u_{, x} & \bar{\varepsilon}_{y}=v_{, y} & \bar{\varepsilon}_{x y}=u_{, y}+v_{, x} \\
k_{x}=-w_{, x x} & k_{y}=-w_{, y y} & k_{x y}=-2 w_{, x y}
\end{array}
$$

In order to obtain the equilibrium equations of rectangular micro plate, the principle of minimum total potential energy is utilized. Hence,

$$
\delta W=\int_{V}\left[\sigma_{x x} \delta \varepsilon_{x x}+\sigma_{y y} \delta \varepsilon_{y y}+\sigma_{x y} \delta \gamma_{x y}\right] d V-\int_{\Omega}\left(P_{x} \delta\left(\frac{\partial w}{\partial x}\right)^{2}+P_{y} \delta\left(\frac{\partial w}{\partial y}\right)^{2}\right) d A=0
$$

So the resulting equilibrium equations for a micro plate are

$$
\begin{aligned}
& \delta u_{x}: N_{x, x}+N_{x y, y}=0 \\
& \delta v_{y}: N_{x y, x}+N_{y, y}=0 \\
& \delta w: M_{x, x x}+2 M_{x y, x y}+M_{y, y y}=P_{x} \frac{\partial^{2} w}{\partial x^{2}}+P_{y} \frac{\partial^{2} w}{\partial y^{2}}
\end{aligned}
$$

In the mentioned equations, $P_{i}$ is buckling load, $N_{i}$ and $M_{i}(i=x, y, x y)$ are force and moment resultants which are obtained by integration of corresponding stress components over the thickness, and are defined as

$$
\left(N_{i}, M_{i}\right)=\int_{-h / 2}^{h / 2} \sigma_{i}(1, z) d z \quad, \quad i=x, x y, y
$$

In determining the force and moment resultants, constitutive equations are needed to define stresses in terms of strain components.

In study of micro-plates, scale of dimensions and effect of micro-structure on the behavior of microplates are important; hence higher order theories are usually used for modeling the micro-structures. In the present study, strain gradient theory is used for modeling the effects of micro scale on relations. Therefore, stress and strain are related not only as the classic form but also considering the gradient of strain. Hence, stress and strain components are related as

$$
\begin{aligned}
& \sigma_{x}=\frac{E(z)}{1-v^{2}}\left(\varepsilon_{x}+v \varepsilon_{y}\right)-g^{2} \frac{E(z)}{1-v^{2}} \nabla^{2}\left(\varepsilon_{x}+v \varepsilon_{y}\right) \\
& \sigma_{y}=\frac{E(z)}{1-v^{2}}\left(\varepsilon_{y}+v \varepsilon_{x}\right)-g^{2} \frac{E(z)}{1-v^{2}} \nabla^{2}\left(\varepsilon_{y}+v \varepsilon_{x}\right) \\
& \sigma_{x y}=\frac{E(z)}{1+v}\left(\varepsilon_{x y}\right)-g^{2} \frac{E(z)}{1+v} \nabla^{2}\left(\varepsilon_{x y}\right)
\end{aligned}
$$


where $\nabla^{2}$ is the two dimensional Laplacian operator in Cartesian coordinate $\left(\nabla^{2}=\frac{\partial^{2}}{\partial x^{2}}+\frac{\partial^{2}}{\partial y^{2}}\right)$. Furthermore, in Eq. (9), the parameter $E(z)$ represents the elastic modulus and $v$ is the Poisson ratio. Here, the "classic" expression refers to the state of length scale parameter equals zero $(g=0)$. Since it is supposed that the micro-plate is made out of functionally graded materials (FGMs), the material properties are function of thickness variable. Thus, let material properties such as elasticity modulus modeled by the power law function through the thickness as

$$
E(z)=E_{m}+E_{c m}(1 / 2-z / h)^{n} \quad E_{c m}=E_{c}-E_{m}
$$

where subscripts $c$ and $m$ refer to the ceramic and metal components, respectively. Moreover, $h$ is plate's thickness $(-h / 2<z<h / 2)$ and $n$ is known as FGM index $(n \geq 0)$. In addition, it was shown that variation of Poisson ratio with respect to the coordinates is not significant in FGMs, so $v$ is supposed to be constant (Mohammadi et al. (2010)).

Substituting Eqs. (10) and (11) in Eqs. (9) and simplifying the results yields the following relation for force and moment resultants

$$
\left[\begin{array}{l}
N_{x} \\
N_{y} \\
N_{x y} \\
M_{x} \\
M_{y} \\
M_{x y}
\end{array}\right]=\left[\begin{array}{cccccc}
A_{11} & A_{12} & 0 & B_{11} & B_{12} & 0 \\
A_{12} & A_{22} & 0 & B_{12} & B_{22} & 0 \\
0 & 0 & A_{33} & 0 & 0 & B_{33} \\
C_{11} & C_{12} & 0 & D_{11} & D_{12} & 0 \\
C_{12} & C_{22} & 0 & D_{12} & D_{22} & 0 \\
0 & 0 & C_{33} & 0 & 0 & D_{33}
\end{array}\right]\left(\left[\begin{array}{c}
u_{, x} \\
v_{, y} \\
u_{, y}+v_{, x} \\
w_{, x x} \\
w_{, y y} \\
w_{, x y}
\end{array}\right]-g^{2} \nabla^{2}\left[\begin{array}{c}
u_{, x} \\
v_{, y} \\
u_{, y}+v_{, x} \\
w_{, x x} \\
w_{, y y} \\
w_{, x y}
\end{array}\right]\right)
$$

where the components of the matrix, shown in Eq. (12), are

$$
\begin{aligned}
& A_{11}=A_{22}=\int_{-h / 2}^{h / 2} \frac{E(z)}{1-v^{2}} d z, A_{12}=\int_{-h / 2}^{h / 2} \frac{v E(z)}{1-v^{2}} d z, A_{33}=\int_{-h / 2}^{h / 2} \frac{E(z)}{2(1+v)} d z \\
& C_{11}=C_{22}=-B_{11}=-B_{22}=\int_{-h / 2}^{h / 2} \frac{z E(z)}{1-v^{2}} d z, C_{12}=-B_{12}=\int_{-h / 2}^{h / 2} \frac{z v E(z)}{1-v^{2}} d z, C_{33}=-\frac{B_{33}}{2}=\int_{-h / 2}^{h / 2} \frac{z E(z)}{2(1+v)} d z \\
& D_{11}=D_{22}=-\int_{-h / 2}^{h / 2} \frac{z^{2} E(z)}{1-v^{2}} d z, D_{12}=-\int_{-h / 2}^{h / 2} \frac{z^{2} v E(z)}{1-v^{2}} d z, D_{33}=-\int_{-h / 2}^{h / 2} \frac{z^{2} E(z)}{(1+v)} d z
\end{aligned}
$$

Substituting Eqs. (12) into Eqs. (8) leads to the governing equilibrium equations. Following the same procedure as was explained in by Javaheri and Eslami (2002) the stability equation for buckling analysis of FG micro-plate is obtained for a rectangular plate as

$$
D^{*} \nabla^{4} w-g^{2} D^{*} \nabla^{6} w=P_{x} w_{, x x}+P_{y} w_{, y y}
$$

where

$$
D^{*}=\frac{B_{11}^{2}-A_{11} D_{11}}{A_{11}}
$$


is interpreted as the equivalent flexural rigidity of functionally graded micro-plate. It is clear that the stability Eq. (14) is changed to the classic form of stability equation for macro-plates by setting the length scale parameter to zero.

\section{NAVIER SOLUTION}

Having an analytical methodology, Navier solution is considered. Therefore, it is assumed that micro-plate is simply supported along all edges.

By using the principle of minimum potential energy and variational approach, the out of-plane boundary are determined as

$$
\oint_{\Gamma}\left(V_{n} \delta w+M_{n} \delta w_{, n}+M_{n n} \delta w_{, n n}\right) d \Gamma=0
$$

where $\Gamma$ denotes the bounds of micro-plate. Also, $M_{n}, M_{n n}$ and $V_{n}$ are called bending moment, higher order bending moment and effective shear force.

Equivalent form of boundary conditions for simply supported edges parallel to the $x$ axis are determined as

$$
\begin{aligned}
& w=w(x, y)=0 \\
& M_{x}=-D^{*}\left(w_{, x x}+v w_{, y y}\right)+g^{2} D^{*}\left(w_{, x x x x}+v w_{, y y y y}+(3-v) w_{, x x y y}\right)=0 \\
& M_{x x}=-g^{2} D^{*}\left(w_{, x x x}+v w_{, x y y}\right)
\end{aligned}
$$

It should be noted that, in the above conditions, the first and second equations are called classical boundary conditions, while the third one is called non-classical boundary conditions.

Based on the Navier solution, the deflection function is assumed as

$$
w(x, y)=\sum_{m=1}^{\infty} \sum_{n=1}^{\infty} W_{m n} \sin (\alpha x) \sin (\beta y) \quad \alpha=(m \pi x / a) \quad, \beta=(n \pi x / b)
$$

It is easy to show that, solution (18) satisfies the prescribed classic and non-classic boundary conditions (17).

Also, for solving the stability equations, pre-buckling forces are needed which are determined from the equilibrium conditions. Since the micro-plate is subjected to uniform in-plane compressive distributed load, the pre-buckling forces are constant and obtained as $P_{x}=P_{y}=-R P$, where $R$ is load parameter. Load parameter $R$ identifies the loading conditions, e.g. micro-plate is loaded uniaxially if $R=0$, subjected to biaxial compressive load if $R=1$ and biaxial compressive and tensile load if $R=-1$.

Upon substituting Eqs. (18) in Eq. (14) and simplifying the resulted equation, the buckling load $P$ is obtained as

$$
P=\frac{D^{*} \pi^{2}\left(m^{4} a^{2} b^{6}+2 m^{2} n^{2} a^{4} b^{4}+n^{4} a^{6} b^{2}+g^{2} m^{6} \pi^{2} b^{6}+3 g^{2} m^{4} \pi^{2} n^{2} a^{2} b^{4}+3 g^{2} m^{2} \pi^{2} n^{4} a^{4} b^{2}+g^{2} n^{6} \pi^{2} a^{6}\right)}{a^{4} b^{4}\left(R n^{2} a^{2}+m^{2} b^{2}\right)}
$$


Eq. (19) gives the buckling load for functionally graded micro-plates where the critical buckling load is the minimum buckling load. It is clear that micro-structure effect influence the buckling load. Moreover considering the size-effects changes the critical buckling load.

\section{NUMERICAL RESULTS AND DISCUSSION}

For having a numerical study and investigation, it is assumed that FG micro-plate is made of silicon-carbide as the ceramic part $\left(E_{c}=420 G P a\right)$ and aluminum as metal part $\left(E_{m}=70 G P a\right)$. Also, the Poisson ratio is constant and equal to $v=0.3$.

To keep the generality of study, results are presented in non-dimensional form. Therefore, the non-dimensional buckling load of micro-plate is defined as

$$
\bar{P}=\frac{P b^{2}}{D_{c}}
$$

where $P$ is the buckling load, $D_{c}$ is the equivalent flexural rigidity based on the ceramic part $(n=0)$ and $b$ is the length of micro-plate.

In table 1, the non-dimensional critical buckling load is tabulated versus different aspect ratios, micro-structure parameters and loading conditions. As table shows, increasing the index of FGM decreases the non-dimensional critical buckling load. Also, increasing the micro-structure parameter increases the buckling load.

\begin{tabular}{|c|c|c|c|c|c|}
\hline$a / b$ & $g / b$ & $n$ & $R=0$ & $R=1$ & $R=-1$ \\
\hline \multirow{6}{*}{0.5} & \multirow{3}{*}{0.1} & 0 & 92.122 & 73.697 & 122.83 \\
\hline & & 1 & 44.597 & 35.678 & 59.466 \\
\hline & & 2 & 34.067 & 27.253 & 45.423 \\
\hline & \multirow{3}{*}{0.33} & 0 & 393.17 & 314.54 & 524.25 \\
\hline & & 1 & 190.34 & 152.27 & 253.80 \\
\hline & & 2 & 145.40 & 116.31 & 193.86 \\
\hline \multirow{6}{*}{1} & \multirow{3}{*}{0.1} & 0 & 47.273 & 23.636 & $122.84^{*}$ \\
\hline & & 1 & 22.883 & 23.636 & $59.465^{*}$ \\
\hline & & 2 & 17.480 & 8.7396 & $45.423^{*}$ \\
\hline & \multirow{3}{*}{0.33} & 0 & 124.35 & 62.172 & $524.25^{*}$ \\
\hline & & 1 & 60.192 & 30.096 & $253.79^{*}$ \\
\hline & & 2 & 45.980 & 22.989 & $193.86^{*}$ \\
\hline \multirow{6}{*}{1.5} & \multirow{3}{*}{0.1} & 0 & 52.937 & 16.288 & $122.84^{*}$ \\
\hline & & 1 & 25.627 & 7.8851 & $59.466^{*}$ \\
\hline & & 2 & 19.577 & 6.0235 & $45.427^{*}$ \\
\hline & \multirow{3}{*}{0.33} & 0 & 118.27 & 36.390 & $390.23^{*}$ \\
\hline & & 1 & 57.255 & 17.616 & $188.91^{*}$ \\
\hline & & 2 & 43.737 & 13.457 & $144.31^{*}$ \\
\hline
\end{tabular}

* Indicates higher mode of buckling.

Table 1: Non-dimensional critical buckling load $\left(\bar{P}^{*} 10^{3}\right)$ for functionally graded micro-plate. 
In Figure 2, the effect of micro-structure parameter on the non-dimensional critical buckling load is plotted for different loading conditions. It is clear that raising the micro-structure parameter is resulted in increasing the non-dimensional critical buckling load. The difference is more apparent as the micro parameter involved in equations. Also, load carrying capacity is more significant for the case of $R=-1$ in comparison with the other cases.

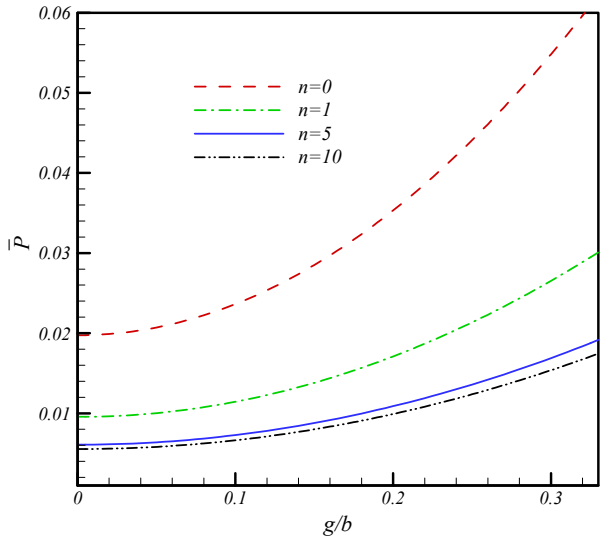

a) Biaxial compressive load $(R=1)$

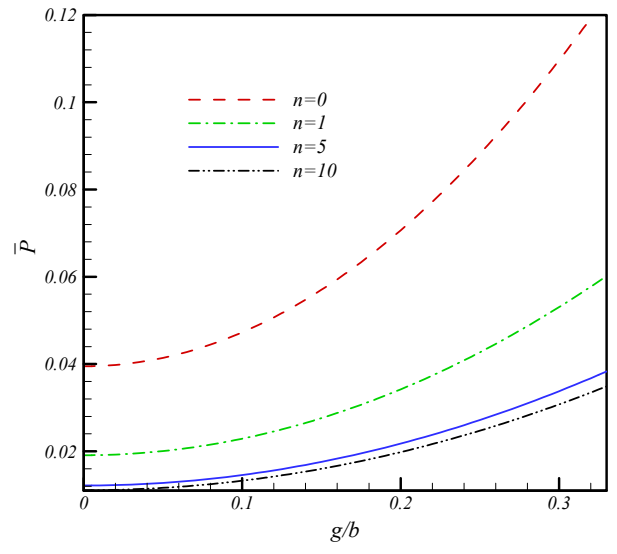

b) Uniaxial compressive load $(R=0)$

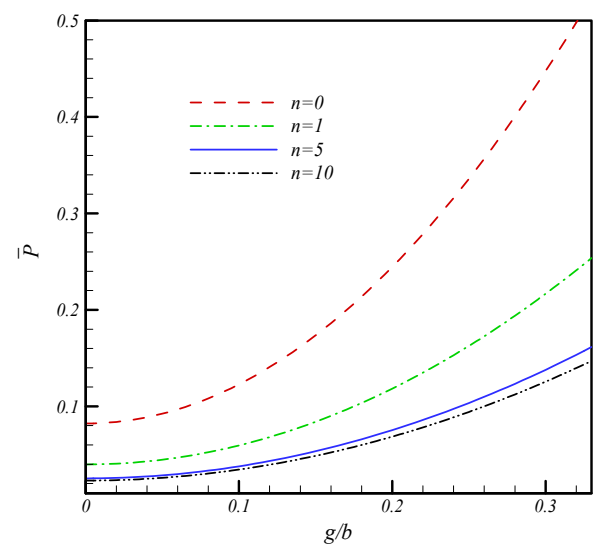

c) Biaxial compressive and tensile load $(R=-1)$

Figure 2: Non-dimensional critical buckling load versus the microstructure parameter.

Variation of buckling load versus the material properties is depicted in Figure 3. As shown in the figure, increasing the index of FGM diminishes the critical buckling load. This refers to increasing the portion of metal component in comparison with the ceramic component in FG composition. Therefore, micro-plate with higher portion of metal has lower flexural rigidity and simply buckle. Also as it is illustrated in this figure, loading conditions affect the critical buckling load. Presence of tensile load, severely increase the load carrying capacity where biaxial compressive load, drops the buckling load.

In Figure 4, the effect of aspect ratio $(a / b)$ on the non-dimensional critical buckling load is presented. Since the critical buckling load corresponds to the smallest possible buckling load, there- 
fore micro-plate may buckle in different mode. As Figure 4 shows, the buckling mode depends on different parameters. It is indicated that loading conditions influence on the buckling mode. So that, micro-plate, subjected to biaxial compressive load, regardless of aspect ratio, always buckles in the first mode. In the case of $R=0$ and $R=-1$, buckling mode depends on the aspect ratio. Increasing the aspect ratio causes the micro-plate buckle in higher modes.

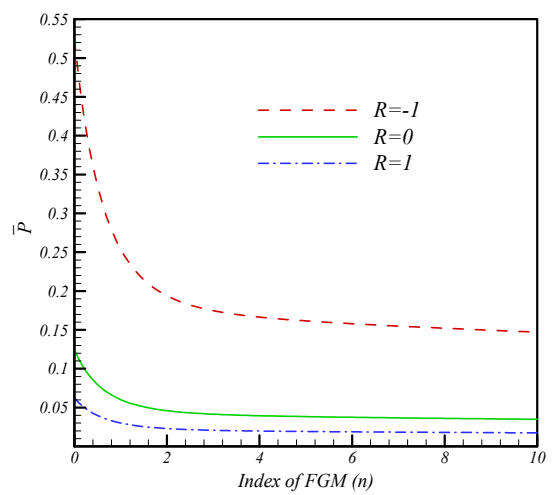

Figure 3: Variation of non-dimensional critical buckling load versus the index of FGM and different loading conditions.

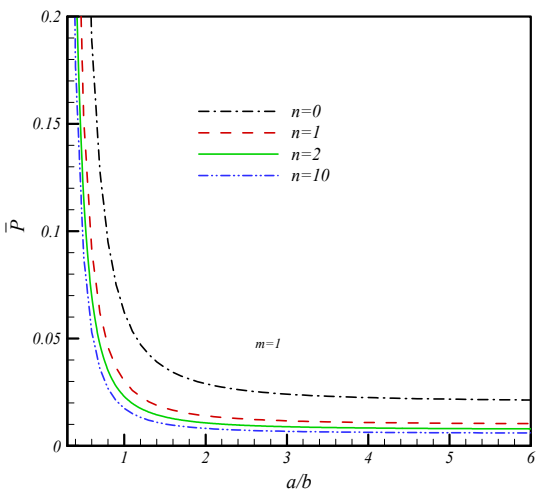

a) Biaxial compressive load $(R=1)$

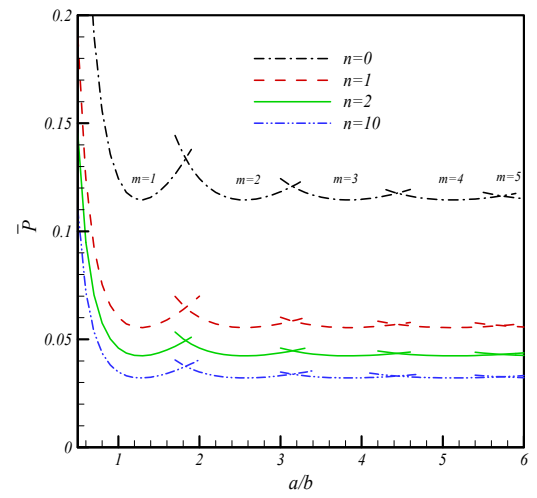

b) Uniaxial compressive load $(R=0)$

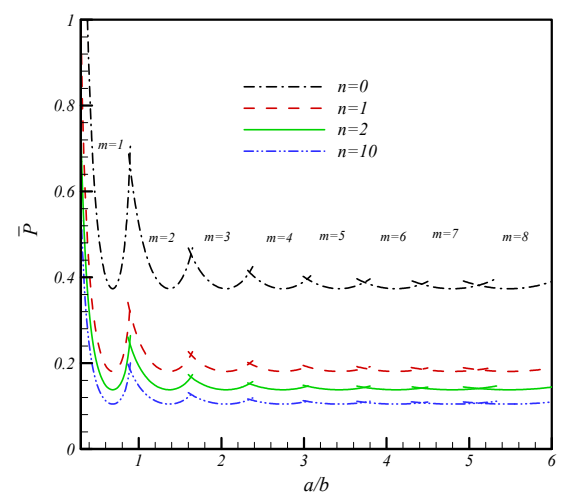

c) Biaxial compressive and tensile load $(R=-1)$

Figure 4: Variation of non-dimensional critical buckling load with respect to aspect ratio. 
In Figure 5, the effect of length scale parameter on the critical buckling load is presented. According to the figure, it is clear that depending on the loading conditions and aspect ratio, microplate may buckle in different modes. Also it is inferred that in each aspect ratio, increasing the length scale parameter postpone the buckling mode.

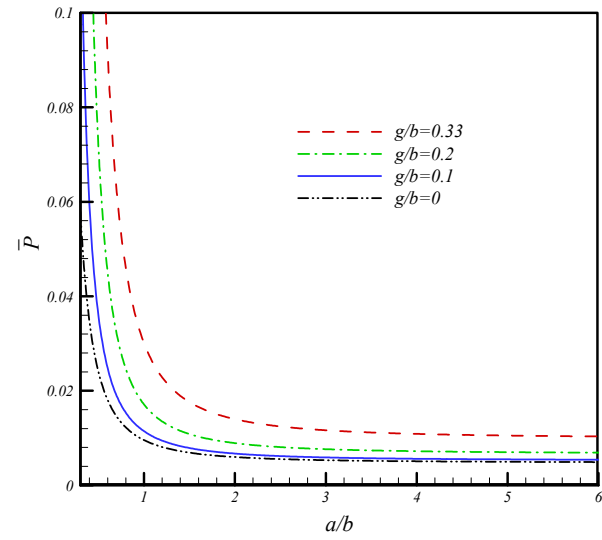

a) Biaxial compressive load $(R=1)$

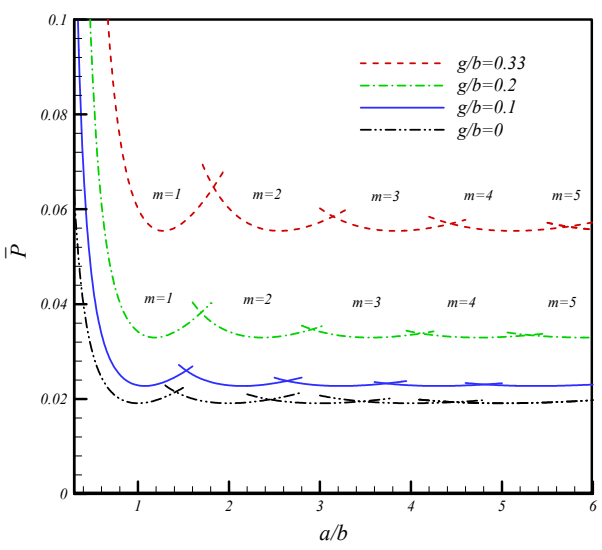

b) Uniaxial compressive load $(R=0)$

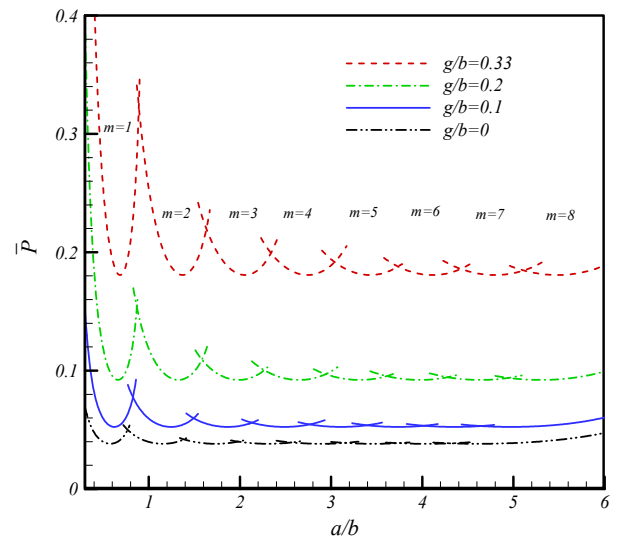

c) Biaxial compressive and tensile load $(R=-1)$

Figure 5: Effect of length scale parameter on the critical buckling mode.

Different mode shapes are shown in Figure 6.

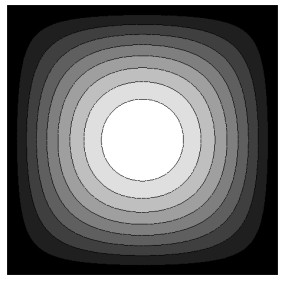

$m=1$

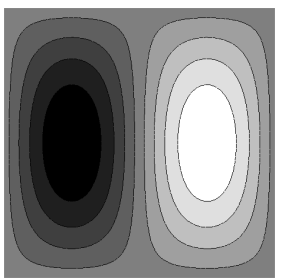

$m=2$

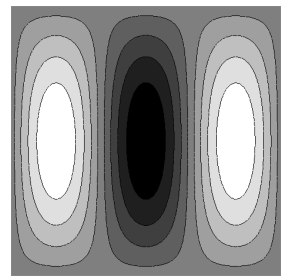

$m=3$

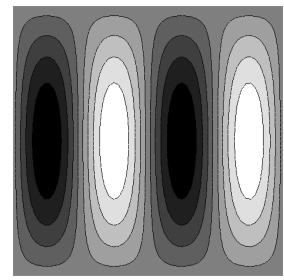

$m=4$

Figure 6: Different buckling mode shapes. 


\section{CONCLUSION}

In the present study, by considering strain gradient theory, buckling analysis of thin rectangular functionally graded micro-plates was surveyed. Using variational approach and principle of minimum total potential energy, higher order governing equations were determined which contain the microstructure parameters. It was assumed micro-plate is made of functionally graded material with power law distribution of material properties through the thickness. Finally, the stability equation was solved analytically for a simply supported micro-plate and critical buckling loads were obtained. It was concluded that increasing the index of FGM decrease the non-dimensional critical buckling load. Also, increasing the microstructure parameter decreases the buckling load. It was inferred that load carrying capacity is greatly depends on the loading conditions. Accordingly, presence of tensile load increases the load capacity. In addition, buckling may occur in higher modes, where the mode is affected by the aspect ratio or loading conditions.

\section{References}

Aifantis, E. (1999) Strain gradient interpretation of size effects. International Journal of Fracture. 95: 299-314.

Asghari, M., Rahaeifard, M., Kahrobaiyan, M.H., Ahmadian, M.T. (2011) The modified couple stress functionally graded Timoshenko beam formulation. Materials and Design 32: 1435-1443.

Chong, A.C.M., Lam, D.C.C. (1999) Strain gradient plasticity effect in indentation hardness of polymers. J. Mater. Res. 14: 4103-4110.

Eringen, A.C. (1966) Linear theory of micro-polar elasticity. J. Math. Mech. 15: 909-923.

Eringen, A.C. (1967) Theory of micro-polar plates. Z. Angew. Math. Phys. 18: 12-30.

Farahmand, H., Ahmadi A.R., Arabnejad, S. (2011) Thermal Buckling analysis of rectangular flexural micro plates using higher continuity p-version finite element method. Thin-walled structures. 49: 1584-1591.

Farahmand, H., Arabnejad, S. (2010) Developing a novel finite elastic approach in strain gradient theory for microstructures. Int. journal for multiscale computational engineering 8: 441-446.

Hofstetter, K., Hellmich, C., Eberhardsteiner, J. (2005) Development and experimental validation of a continuum micromechanics model for the elasticity of wood. Eur. J. Mech. A/Solids. 24: 1030-1053.

Javaheri, R., Eslami, M.R. (2002) Buckling of functionally graded plates under in-plane compressive loading. ZAMM. Z. Angew. Math. Mech. 82: 277-28.

Jayakumar, T., Sandhya, R., Rao, B.P.C., Bhaduri, A.K., Ramu, I., Mohanty, S.C. (2014) Structural Integrity Buckling Analysis of Rectangular Functionally Graded Material Plates under Uniaxial and Biaxial Compression Load. Procedia Engineering, 86: 748-757.

Ke, L.L., Wang, Y.S. (2011) Size effect on dynamic stability of functionally graded micro-beams based on a modified couple stress theory. Composite Structures 93: 342-350.

Ke, L.L., Wang, Y.S., Yang, J. (2012) Kitipornchai, S., Nonlinera free vibration of size dependent functionally graded micro-beams. International Journal of Engineering Science 50: 256-267.

Koizumi, M. (1997) FGM activities in Japan. Composites 28: 1-4.

Lakes, R.S. (1980) The role of gradient effects in the piezoelectricity of bone. IEEE Trans.Biomed. Eng., BME. 27: 282-283.

Lakes, R.S. (1986) Experimental microelasticity of two porous solids. Int. J. Solids Struc. 22: 55-63.

Lakes, R.S. (1995) Experimental methods for study of Cosserat elastic solids and other generalized elastic continua, Continuum Models for Materials with Microstructure, H.B. Mhlhaus (ed.), Wiley, Chichester. 
Lazopoulos, K.A. (2009) On bending of strain elastic micro-plates. Mechanics Research Communications 36: 777-783. Mindlin, R.D. (1964) Micro-structure in linear elasticity. Arch. Rat. Mech. Anal. 16: 51-78.

Mindlin, R.D., Eshel, N.N. (1986) On first strain-gradient theories in linear elasticity, Int. J. Solids Struct. 4: 109124.

Mohammadi, M., Saidi, A.R., Jomehzadeh, E. (2009) A novel analytical approach for buckling analysis of moderately thick functionally graded rectangular plates with two simply supported opposite edges. Proc. IMechE Part C: J. Mechanical Engineering Science 224: 1831-1841.

Mohammadi, M., Saidi, A.R., Jomehzadeh, E. (2010) Levy solution for buckling analysis of functionally graded rectangular plates. Appl. Compos. Mater. 17: 81-93.

Papargyri-Beskou, S., Beskos, D.E. (2008) Static, stability and dynamic analysis of gradient elastic flexural Kirchhoff plates. Arch. Appl. Mech. 78: 625-635.

Thinh, T. I., Tu, T.M., Quoc, T.H., Long, N.V. (2016) Vibration and Buckling Analysis of Functionally Graded Plates Using New Eight-Unknown Higher Order Shear Deformation Theory. Lat. Am. j. solids struct. 13: 456-477.

Tiersten, H.F., Bleustein, J.L. (1974) Generalized elastic continua, G. Herrmann, R.D. Mindlin (eds.), and Applied Mechanics. Elsevier Ltd. New York.

Toupin, R.A. (1962) Elasic materials with couple stress. Arch. Ration. Mech. Anal. 11: 385-414.

Vardoulakis, I., Sulem, J. (1995) Bifurcation Analysis in Geomechanics. Chapman and Hall, London.

Wang, B., Zhou, S., Zhao J., Chen, X. (2011) A size dependent Kirchhoff micro-plate model based on strain gradient elasticity theory. European Journal of Mechanics A/Solids 30: 517-524.

Wang, C.M., Reddy, J.N., Lee, K.H. (2000) Shear deformable beams and plates, relationship with the classical theory, Elsevier Ltd. New yourk.

Wu, C.P., Chen Y.C., Peng, S.T. (2013) Buckling analysis of functionally graded material circular hollow cylinders under combined axial compression and external pressure. Thin-Walled Structures. 69: 54-66.

Yang, F., Chong, A.C.M., Lam, D.C.C., Tong, P. (2002) Couple stress based strain gradient theory for elasticity. Int. J.Solids Struct. 39: 2731-2743.

Yang, J.F.C., Lakes, R.S. (1982) Experimental study of micropolar and couple stress elasticity in compact bone in bending. J. Biomech. 1: 91-98. 